\title{
LEFT COUNITAL HOPF ALGEBRAS ON FREE NIJENHUIS ALGEBRAS
}

\author{
XING GAO, PENG LEI, AND TIANJIE ZHANG*
}

\begin{abstract}
AвstRact. Factorization in algebra is an important problem. In this paper, we first obtain a unique factorization in free Nijenhuis algebras. By using of this unique factorization, we then define a coproduct and a left counital bialgebraic structure on a free Nijenhuis algebra. Finally, we prove that this left counital bialgebra is connected and hence obtain a left counital Hopf algebra on a free Nijenhuis algebra.
\end{abstract}

\section{CONTENTS}

1. Introduction

2. Left counital bialgebra structures on free Nijenhuis algebras

2.1. A free Nijenhuis algebras on a set

2.2. The left counital bialgebra structure on free Nijenhuis algebras

3. Left counital Hopf algebra structures on free Nijenhuis algebras

References

\section{INTRODUCTION}

A Nijenhuis algebra $(R, N)$ is an associative algebra $R$ equipped with a linear operator $N$ : $R \rightarrow R$, called Nijenhuis operator, satisfying the Nijenhuis equation:

$$
N(u) N(v)=N(N(u) v)+N(u N(v))-N^{2}(u v) \text { for all } u, v \in R .
$$

The Lie algebra version of the associative Nijenhuis equation started in earnest in the 1950s [18]. In that paper, Nijenhuis introduced the crucial concept of Nijenhuis tensor, which fits closely into the distinguished concepts of Schouten-Nijenhuis bracket, the Frölicher-Nijenhuis bracket [8] and the Nijenhuis-Richardson bracket. Thereafter, Magri et at. [15] studied the deformation of Lie brackets given by Nijenhuis operators. Recently, Sokolov et al. [10, 11] examined the Nijenhuis operators on Lie algebras in the context of the classical Yang-Baxter equation, which has a close relation with the Lie algebraic version of the Rota-Baxter equation recalled below [4, 13].

The Nijenhuis operator on an associative algebra can be originated to [5] in the study of quantum bi-Hamiltonian systems. In [7], K. Ebrahimi-Fard gave the construction of a free commutative associative Nijenhuis algebra on a commutative associative algebra, based on an augmented modified quasi-shuffle product. In [20], the associative Nijenhuis operators were constructed due to an application of a twisting operation on Hochschild complex by analogy with Drinfelds

Date: November 9, 2018.

2010 Mathematics Subject Classification. 16W99, 08B20 16T10 16T05 16T30 .

Key words and phrases. Nijenhuis algebra, bracketed words, factorization, left counital bialgebra, left counital Hopf algebra.

*Corresponding author. 
twisting operations. Latterly, Guo et al. constructed the free noncommutative associative Nijenhuis algebra on an algebra, and studied the associative Nijenhuis algebras with emphasis on the relationship between the category of Nijenhuis algebras and the category of NS algebras [16].

Hopf algebras, named after Heinz Hopf, occur naturally in algebraic topology, where they have broad connections with many areas in mathematics and mathematical physics [1, 6, 19]. Much of the research in Hopf algebras are on specific classes of examples. A crucial class of Hopf algebras is built from free objects in various contexts, such as free associative algebras, the enveloping algebras of Lie algebras, and the free objects in the category of dendriform algebras of Loday and of tridendriform algebras of Loday and Ronco [17]. It is worth to note that the classical ConnesKreimer Hopf algebra of rooted trees can be considered as a free operated algebra [14, 21].

The Nijenhuis equation can be viewed as the homogeneous version of the familiar Rota-Baxter equation:

$$
P(u) P(v)=P(u P(v))+P(P(u) v)+\lambda P(u v), \text { for all } u, v \in R,
$$

which gives the Rota-Baxter operator $P$ on an associative algebra $R$. Here $\lambda$, called the weight of $P$, is a prefixed element in the base ring of the algebra $R$. See [2, 3, 12, 13] for further details and references. Hopf algebra structures were equipped on free noncommutative Rota-Baxter algebras [21], and Hopf algebra related structures were explored on free commutative Nijenhuis algebras [22]. Following closely these two inspiring works [21, 22], we equip Hopf type algebra related structures on free noncommutative Nijenhuis algebras in this paper, by making use of the construction of free Nijenhuis algebras via bracketed words given in [9, 16].

Here is the layout of this paper. In Section 2 , we review the construction of free noncommutative Nijenhuis algebra on a set in terms of bracketed words, and then acquire a unique factorization of such bracketed words (Proposition 2.5). Using this unique factorization, we define a coproduct on a free Nijenhuis algebra, which, together with a left counity, turns the free Nijenhuis algebra into a left counital cocycle bialgebra (Theorem 2.14), satisfying an one-cocycle condition (Eq. (4). In the final Section 3, we grade the left counital bialgebra obtained in the previous section, and prove that it is connected and hence a left counital Hopf algebra (Theorem 3.6).

Convention. In this paper, all algebras are taken to be unitary associative (but not necessary commutative) over a unitary commutative ring $\mathbf{k}$, unless the contrary is specified. Also linear maps and tensor products are taken over k. For any set $Y$, let $M(Y)$ and $S(Y)$ denote the free monoid and free semigroup generated by $Y$, respectively.

\section{Left counital bialgebra Structures on freE NiJenhUis algebras}

In this section, we give a left counital cocycle bialgebra structure on a free Nijenhuis algebra. We first recall the construction of free Nijenhuis algebras by bracketed words.

\subsection{A free Nijenhuis algebras on a set.}

Definition 2.1. Let $X$ be a set. A free Nijenhuis algebra on $X$ is a Nijenhuis algebra $\left(F_{\mathrm{N}}(X), N_{X}\right)$ together with a map $j_{X}: X \rightarrow F_{\mathrm{N}}(A)$ such that, for any Nijenhuis algebra $(R, N)$ and any map $f: X \rightarrow R$, there is a unique Nijenhuis algebra homomorphism $\bar{f}: F_{\mathrm{N}}(X) \rightarrow R$ such that $\bar{f} \circ j_{X}=f:$

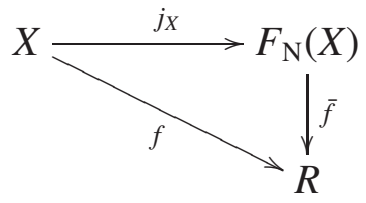


Free Nijenhuis algebras are quotients of free operated algebras, and the construction of free operated algebras was given in [13, 14]. We reproduce that construction here to review the notations.

Let $\lfloor Y\rfloor$ denote the set $\{\lfloor y\rfloor \mid y \in Y\}$. Thus $\lfloor Y\rfloor$ is a set indexed by $Y$ but disjoint with $Y$. For a set $X$, we first let $\mathfrak{M}_{0}:=M(X)$ be the free monoid generated by $X$, where the identity is denoted by 1. Then we define $\mathfrak{M}_{1}:=M(X \cup\lfloor M(X)\rfloor)$ with $i_{0,1}$ being the natural injection

$$
i_{0,1}: \mathfrak{M}_{0}=M(X) \hookrightarrow \mathfrak{M}_{1}=M\left(X \cup\left\lfloor\mathfrak{M}_{0}\right\rfloor\right) .
$$

We identify $\mathfrak{M}_{0}$ with its image in $\mathfrak{M}_{1}$. In particular, the identity 1 in $\mathfrak{M}_{0}$ is sent to 1 in $\mathfrak{M}_{1}$.

Inductively assume that $\mathfrak{M}_{n}$ has been defined for $n \geq 1$, and define

$$
\mathfrak{M}_{n+1}:=M\left(X \cup\left\lfloor\mathfrak{M}_{n}\right\rfloor\right) .
$$

Further assume that the embedding

$$
i_{n-1, n}: \mathfrak{M}_{n-1} \rightarrow \mathfrak{M}_{n}
$$

has been obtained. Then we have the injection

$$
\left\lfloor\mathfrak{M}_{n-1}\right\rfloor \hookrightarrow\left\lfloor\mathfrak{M}_{n}\right\rfloor .
$$

Thus by the freeness of $\mathfrak{M}_{n}=M\left(X \cup\left\lfloor\mathfrak{M}_{n-1}\right\rfloor\right)$, we have

$$
\mathfrak{M}_{n}=M\left(X \cup\left\lfloor\mathfrak{M}_{n-1}\right\rfloor\right) \hookrightarrow M\left(X \cup\left\lfloor\mathfrak{M}_{n}\right\rfloor\right)=\mathfrak{M}_{n+1} .
$$

We finally define the monoid

$$
\mathfrak{M}(X):=\underset{\lim }{\longrightarrow} \mathfrak{M}_{n}=\bigcup_{n \geq 0} \mathfrak{M}_{n}
$$

with identity 1 . Elements of $\mathfrak{M}(X)$ are called bracketed words in $X$. Let $\mathbf{k} \mathfrak{M}(X)$ be the free k-module spanned by $\mathfrak{M}(X)$. Since the basis is a monoid, the multiplication on $\mathfrak{M}(X)$ can be extended via linearity to turn the k-module $\mathbf{k M M}(X)$ into an algebra, which we still denote by kMi $(X)$. Similarly, we can extend the operator \lfloor\rfloor$: \mathfrak{M}(X) \rightarrow \mathfrak{M}(X)$, which takes $w \in \mathfrak{M}(X)$ to $\lfloor w\rfloor$, to an operator \lfloor\rfloor on $\mathbf{k} \mathfrak{M}(X)$ by linearity and turn the algebra $\mathbf{k M}(X)$ into an operated algebra.

Lemma 2.2. [13, 14] Let $i_{X}: X \rightarrow \mathfrak{M}(X)$ and $j_{X}: \mathfrak{M}(X) \rightarrow \mathbf{k} \mathfrak{M}(X)$ be the natural embeddings. Then, with structures as above,

(a) the triple $\left(\mathfrak{M}(X),\llcorner\rfloor, i_{X}\right)$ is the free operated monoid on $X$; and

(b) the triple $\left(\mathbf{k M M}(X),\lfloor\rfloor, j_{X} \circ i_{X}\right)$ is the free operated algebra on $X$.

It was shown in [13] that every $w \in \mathfrak{M}(X) \backslash\{\mathbf{1}\}$ has a unique standard decomposition:

$$
w=w_{1} \cdots w_{m},
$$

where $w_{i}, 1 \leq i \leq m$, is alternatively in the free semigroup $S(X)$ or in $\lfloor\mathfrak{M}(X)\rfloor:=\{\lfloor u\rfloor \mid u \in \mathfrak{M}(X)\}$. We call $m$ the breadth of $w$, denoted by bre $(w)=m$. If $w=\mathbf{1} \in \mathfrak{M}(X)$, we define bre $(w)=0$. Elements $w \in \mathfrak{M}_{n} \backslash \mathfrak{M}_{n-1}$ are said to have depth $n$, denoted by $\operatorname{dep}(w)=n$.

We now review a k-basis of a free Nijenhuis algebra given in [9, [16], which is a subset of bracketed words. Let $Y$ and $Z$ be subsets of $\mathfrak{M}(X)$. Define the alternating products of $Y$ and $Z$ by

$$
\begin{aligned}
\Lambda(Y, Z)= & \left(\bigcup_{r \geq 1}(Y\lfloor Z\rfloor)^{r}\right) \bigcup\left(\bigcup_{r \geq 0}(Y\lfloor Z\rfloor)^{r} Y\right) \\
& \bigcup\left(\bigcup_{r \geq 1}(\lfloor Z\rfloor Y)^{r}\right) \bigcup\left(\bigcup_{r \geq 0}(\lfloor Z\rfloor Y)^{r}\lfloor Z\rfloor\right) \bigcup\{\mathbf{1}\} .
\end{aligned}
$$


Recursively define

$$
\mathfrak{X}_{0}:=M(X) \text { and } \mathfrak{X}_{n}:=\Lambda\left(\mathfrak{X}_{0}, \mathfrak{X}_{n-1}\right), n \geq 1 \text {. }
$$

Thus $\mathfrak{X}_{0} \subseteq \cdots \subseteq \mathfrak{X}_{n} \subseteq \cdots$ and so we have

$$
\mathfrak{X}_{\infty}:=\lim _{\longrightarrow} \mathfrak{X}_{n}=\bigcup_{n \geq 0} \mathfrak{X}_{n} .
$$

Let $F_{\mathrm{N}}(X):=\mathbf{k} \mathfrak{X}_{\infty}$ the free $\mathbf{k}$-module spanned by $\mathfrak{X}_{\infty}$. To make $F_{\mathrm{N}}(X)$ into a Nijenhuis algebra, a Nijenhuis operator $N_{X}$ and a product $\diamond$ need to be equipped. Let $w, w^{\prime}$ be two basis elements in $\mathfrak{X}_{\infty}$. Define a linear operator $N_{X}: F_{\mathrm{N}}(X) \rightarrow F_{\mathrm{N}}(X)$ by setting

$$
N_{X}(w)=\lfloor w\rfloor \text {. }
$$

Next we define $w \diamond w^{\prime}$ inductively on the $\operatorname{sum} n:=\operatorname{dep}(w)+\operatorname{dep}\left(w^{\prime}\right) \geq 0$. If $n=0$, then $w, w^{\prime} \in \mathfrak{X}_{0}=M(X)$ and define $w \diamond w^{\prime}:=x x^{\prime}$, the concatenation in $M(X)$. Suppose that $w \diamond w^{\prime}$ have been defined for all $w, w^{\prime} \in \mathfrak{X}_{\infty}$ with $n \leq k$ for a $k \geq 0$ and consider $w, w^{\prime} \in \mathfrak{X}_{\infty}$ with $n=k+1$. If $w=\mathbf{1}$ or $w^{\prime}=\mathbf{1}$, without loss of generality, letting $w=\mathbf{1}$, then define $w \diamond w^{\prime}:=w^{\prime}$. Assume $w \neq 1$ and $w^{\prime} \neq \mathbf{1}$, and so $\operatorname{bre}(w), \operatorname{bre}\left(w^{\prime}\right) \geq 1$. Consider first bre $(w)=\operatorname{bre}\left(w^{\prime}\right)=1$. Then $w$ and $w^{\prime}$ are in $S(X) \subset \mathfrak{X}_{0}$ or $\left\lfloor\mathfrak{X}_{\infty}\right\rfloor$ and can not be both in $S(X)$ since $n=k+1 \geq 1$. We accordingly define

$$
w \diamond w^{\prime}= \begin{cases}w w^{\prime}, & \text { if } w \in S(X), w^{\prime} \in\left\lfloor\mathfrak{X}_{\infty}\right\rfloor, \\ w w^{\prime}, & \text { if } w \in\left\lfloor\mathfrak{X}_{\infty}\right\rfloor, w^{\prime} \in S(X), \\ \left\lfloor w \diamond \bar{w}^{\prime}\right\rfloor+\left\lfloor\bar{w} \diamond w^{\prime}\right\rfloor-\left\lfloor\left\lfloor\bar{w} \diamond \bar{w}^{\prime}\right\rfloor\right\rfloor, & \text { if } w=\lfloor\bar{w}\rfloor, w^{\prime}=\left\lfloor\bar{w}^{\prime}\right\rfloor \in\left\lfloor\mathfrak{X}_{\infty}\right\rfloor .\end{cases}
$$

Here the product in the first two cases is by concatenation and in the third case is by the induction hypothesis on $n$. Now consider bre $(w) \geq 1$ or bre $\left(w^{\prime}\right) \geq 1$. Let $w=w_{1} \cdots w_{m}$ and $w^{\prime}=w_{1}^{\prime} \cdots w_{m^{\prime}}^{\prime}$ be the standard decompositions of $w$ and $w^{\prime}$. Define

$$
w \diamond w^{\prime}:=w_{1} \cdots w_{m-1}\left(w_{m} \diamond w_{1}^{\prime}\right) w_{2}^{\prime} \cdots w_{m^{\prime}}^{\prime},
$$

where $w_{m} \diamond w_{1}^{\prime}$ is defined by Eq. (2) and the rest products are given by the concatenation.

Lemma 2.3. [9, 16] Let $X$ be a set and $j_{X}: X \rightarrow \mathfrak{X}_{\infty} \rightarrow F_{\mathrm{N}}(X)$ the natural injection. Then the quadruple $\left(F_{\mathrm{N}}(X), \diamond, N_{X}, j_{X}\right)$ is the free Nijenhuis algebra on $X$.

For the rest of this paper, unless alternative notations are specifically given, we will use the infix notation $\lfloor w\rfloor$ interchangeably with $N_{X}(w)$ for any $w \in F_{\mathrm{N}}(X)$. Write

$$
\mathfrak{J}:=X \sqcup\left\lfloor\mathfrak{X}_{\infty}\right\rfloor \text {. }
$$

Definition 2.4. We call a sequence $w_{1}, \cdots, w_{m}$ from the set $\mathfrak{I}$ alternating if no consecutive elements in the sequence are in $\left\lfloor\mathfrak{X}_{\infty}\right\rfloor$, that is, either $w_{i}$ or $w_{i+1}$ is in $X$ for each $1 \leq i \leq m-1$.

The following unique factorization sheds insight on the construction of a coproduct on $F_{\mathrm{N}}(X)$.

Proposition 2.5. Let $w \in \mathfrak{X}_{\infty} \backslash\{\mathbf{1}\}$. Then there is a unique alternating sequence $w_{1}, \cdots, w_{m}$ in $\mathfrak{I}$ such that

$$
w=w_{1} \diamond \cdots \diamond w_{m}
$$

called the alternating factorization of $w$. We also call $\operatorname{wid}(\mathrm{w}):=\mathrm{m}$ the width of $w$. If $w=\mathbf{1}$, we define $\operatorname{wid}(\mathrm{w}):=0$.

Proof. (Existence). Suppose $w=u_{1} \cdots u_{n}$ is the standard decomposition of $w$. Then $u_{1}, \cdots, u_{n}$ are alternatively in $S(X)$ and $\left\lfloor\mathfrak{X}_{\infty}\right\rfloor$. Expanding the factors that are in $S(X)$, we get

$$
w=w_{1} \cdots w_{m} \text {, where } w_{1}, \cdots, w_{m} \text { is an alternating sequence. }
$$


It follows from Eq. (2) that $w=w_{1} \diamond \cdots \diamond w_{m}$.

(Uniqueness). Suppose $w_{1}, \cdots, w_{m}$ and $w_{1}^{\prime}, \cdots, w_{m^{\prime}}^{\prime}$ are two alternating sequences such that

$$
w=w_{1} \diamond \cdots \diamond w_{m}=w_{1}^{\prime} \diamond \cdots \diamond w_{m^{\prime}}^{\prime}
$$

By Definition 2.4 and Eq. (2), we get

$$
w_{1} \cdots w_{m}=w_{1} \diamond \cdots \diamond w_{m}=w=w_{1}^{\prime} \diamond \cdots \diamond w_{m^{\prime}}^{\prime}=w_{1}^{\prime} \cdots w_{m^{\prime}}^{\prime},
$$

and so $m=m^{\prime}$ and $w_{i}=w_{i}^{\prime}$ for $1 \leq i \leq m$ by the construction of $\mathfrak{X}_{\infty}$.

Remark 2.6. (a) The breadth and width of $w \in \mathfrak{X}_{\infty}$ are different. For example, let $w=x_{1} x_{2} x_{3}$ with $x_{1}, x_{2}, x_{3} \in X$. Then $\operatorname{bre}(w)=1$ and $\operatorname{wid}(w)=3$.

(b) If the sequence $w_{1}, \cdots, w_{m}$ is alternating, then, for each $1 \leq i \leq m-1, w_{i}$ and $w_{i+1}$ cannot be both in $\left\lfloor\mathfrak{X}_{\infty}\right\rfloor$. However, $w_{i}$ and $w_{i+1}$ may be both in $X$.

2.2. The left counital bialgebra structure on free Nijenhuis algebras. In this subsection, we use Proposition 2.5 to get a coproduct on $F_{\mathrm{N}}(X)$, which, together with a left counit, makes $F_{\mathrm{N}}(X)$ into a left counital coalgebra. The 1-cocycle condition $\Delta P=P \otimes \mathbf{1}+(\mathrm{id} \otimes P) \Delta$, which is used to construct the Hopf algebra structure on free Rota-Baxter algebras [21], does not work for free Nijenhuis algebras. So Guo et al. proposed the following concepts [22].

Definition 2.7. (a) A left counital coalgebra is a triple $(H, \Delta, \varepsilon)$, where the coproduct $\Delta$ : $H \rightarrow H \otimes H$ satisfies the coassociativity: $(\Delta \otimes \mathrm{id}) \Delta=(\mathrm{id} \otimes \Delta) \Delta$ and the left counit $\varepsilon: H \rightarrow \mathbf{k}$ satisfies the left counicity: $(\varepsilon \otimes \mathrm{id}) \Delta=\beta_{\ell}$, where $\beta_{\ell}: H \rightarrow \mathbf{k} \otimes H, u \mapsto 1 \otimes u$ for all $u \in H$.

(b) A left counital bialgebra is a quintuple $(H, m, u, \Delta, \varepsilon)$, where $(H, m, u)$ is an algebra and $(H, \Delta, \varepsilon)$ is a left counital coalgebra such that $\Delta$ and $\varepsilon$ are homomorphisms of algebras.

(c) A left counital operated bialgebra is a sextuple $(H, m, u, \Delta, \varepsilon, P)$, where $(H, m, u, \Delta, \varepsilon)$ is a left counital bialgebra and $(H, m, u, P)$ is an operated algebra, that is, an algebra $(H, m, u)$ with a linear operator $P: H \rightarrow H$.

(d) A left counital cocycle bialgebra is a left counital operated bialgebra $(H, m, u, \Delta, \varepsilon, P)$ satisfying the one-cocycle property:

$$
\Delta P=(\mathrm{id} \otimes P) \Delta .
$$

Thanks to Eq. (4), we can construct a coproduct $\Delta: F_{\mathrm{N}}(X) \rightarrow F_{\mathrm{N}}(X) \otimes F_{\mathrm{N}}(X)$ by defining $\Delta(w)$ for $w \in \mathfrak{X}_{\infty}$ through an induction on the $\operatorname{depth} \operatorname{dep}(w)$. If $\operatorname{dep}(w)=0$, then $w \in \mathfrak{X}_{0}=M(X)$. First we define

$$
\Delta(w):=\mathbf{1} \otimes \mathbf{1} \text { provided } w=\mathbf{1}
$$

and

$$
\Delta(w):=\mathbf{1} \otimes x \text { provided } w=x \in X .
$$

If $w=x_{1} \cdots x_{m} \in S(X)$ with $m \geq 2$ and $x_{i} \in X$ for $1 \leq i \leq m$, then $w=x_{1} \diamond \cdots \diamond x_{m}$ by Eq. (2), and we define

$$
\Delta(w):=\Delta\left(x_{1}\right) \diamond \cdots \diamond \Delta\left(x_{m}\right) .
$$

Assume $\Delta(w)$ has been defined for $w \in \mathfrak{X}_{\infty}$ with $\operatorname{dep}(w) \leq n$ and consider $w \in \mathfrak{X}_{\infty}$ with $\operatorname{dep}(w)=n+1$. In view of Proposition 2.5, there is a unique alternating sequence $w_{1}, \cdots, w_{m}$ such that

$$
w=w_{1} \diamond \cdots \diamond w_{m}, \text { where } w_{1}, \cdots, w_{m} \in \mathfrak{J}, m \geq 1
$$


If $m=1$, then $w \in\left\lfloor\mathfrak{X}_{\infty}\right\rfloor$ by $\operatorname{dep}(w)=n+1 \geq 1$. So we can write $w:=\lfloor\bar{w}\rfloor$ for some $\bar{w} \in \mathfrak{X}_{\infty}$. Then we define by Eq. (4) that

$$
\Delta(w):=\Delta(\lfloor\bar{w}\rfloor):=\left(\mathrm{id} \otimes N_{X}\right) \Delta(\bar{w})
$$

where $\Delta(\bar{w})$ is defined by the induction hypothesis. If $m \geq 2$, we define

$$
\Delta(w):=\Delta\left(w_{1}\right) \diamond \cdots \diamond \Delta\left(w_{m}\right)
$$

where $\Delta\left(w_{1}\right), \cdots, \Delta\left(w_{m}\right)$ are defined in Eq. (6) or Eq. (8). Note $\Delta(w)$ is well-defined by the uniqueness of the alternating factorization of $w$ in Proposition 2.5. This completes the inductive definition of the coproduct $\Delta$ on $F_{\mathrm{N}}(X)$. We shall tacitly denote by $\Delta$ the coproduct defined here throughout the remainder of the paper.

The following is an easy property of $\Delta$, which will be used frequently.

Lemma 2.8. Let $X$ be a set and $w \in M(X)$. Then $\Delta(w)=\mathbf{1} \otimes w$.

Proof. Consider first $w=\mathbf{1}$. Then $\Delta(w)=\mathbf{1} \otimes \mathbf{1}=\mathbf{1} \otimes w$ by Eq. (5). Consider next $w \neq \mathbf{1}$. Then we may write $w=x_{1} \cdots x_{m}$, where $m \geq 1$ and $x_{1}, \cdots, x_{m} \in X$. Then it follows from Eqs. (6) and (7) that

$$
\begin{aligned}
\Delta(w) & =\Delta\left(x_{1} \cdots x_{m}\right)=\Delta\left(x_{1}\right) \diamond \cdots \diamond \Delta\left(x_{m}\right) \\
& =\left(\mathbf{1} \otimes x_{1}\right) \diamond \cdots \diamond\left(\mathbf{1} \otimes x_{m}\right)=\mathbf{1} \otimes\left(x_{1} \diamond \cdots \diamond x_{m}\right) \\
& =\mathbf{1} \otimes\left(x_{1} \cdots x_{m}\right)=\mathbf{1} \otimes w,
\end{aligned}
$$

as required.

We are going to show the compatibility of $\Delta$ with the product $\diamond$. Let us begin with a simple case.

Lemma 2.9. Let $X$ be a set and $u, v \in M(X)$. Then

$$
\Delta(u \diamond v)=\Delta(u) \diamond \Delta(v)
$$

Proof. We have two cases to consider.

Case 1. $u=1$ or $v=1$. By symmetry, let $u=1$. Then $\Delta(u)=\mathbf{1} \otimes \mathbf{1}$ by Eq. (5) and so

$$
\Delta(u \diamond v)=\Delta(v)=(\mathbf{1} \otimes \mathbf{1}) \diamond \Delta(v)=\Delta(u) \diamond \Delta(v) .
$$

Case 2. $u \neq \mathbf{1}$ and $v \neq \mathbf{1}$. Then we can write

$$
u=x_{1} \cdots x_{p} \text { and } v=y_{1} \cdots y_{q},
$$

where $p, q \geq 1$ and $x_{1}, \cdots, x_{p}, y_{1}, \cdots, y_{q} \in X$. So

$$
\begin{aligned}
\Delta(u \diamond v) & =\Delta\left(x_{1} \cdots x_{p} y_{1} \cdots y_{q}\right) \quad(\text { by Eq. (2) }) \\
& =\mathbf{1} \otimes\left(x_{1} \cdots x_{p} y_{1} \cdots y_{q}\right) \quad(\text { by Lemma 2.8) } \\
& \left.=\mathbf{1} \otimes\left(\left(x_{1} \cdots x_{p}\right) \diamond\left(y_{1} \cdots y_{q}\right)\right) \quad \text { (by Eq. (3) }\right) \\
& =\left(\mathbf{1} \otimes\left(x_{1} \cdots x_{p}\right)\right) \diamond\left(\mathbf{1} \otimes\left(y_{1} \cdots y_{q}\right)\right) \\
& =\Delta\left(x_{1} \cdots x_{p}\right) \diamond \Delta\left(y_{1} \cdots y_{q}\right) \quad(\text { by Lemma 2.8) } \\
& =\Delta(u) \diamond \Delta(v),
\end{aligned}
$$

as required. 
Lemma 2.10. Let $X$ be a set and $u, v \in F_{\mathrm{N}}(X)$. Then

$$
\Delta(u \diamond v)=\Delta(u) \diamond \Delta(v) .
$$

Proof. Since $\Delta$ is linear and $\diamond$ is bilinear, we only need to prove the result for basis elements $u, v \in \mathfrak{X}_{\infty}$. If $u=\mathbf{1}$ or $v=\mathbf{1}$, without loss of generality, letting $u=\mathbf{1}$, then by Eq. (5),

$$
\Delta(u \diamond v)=\Delta(v)=(\mathbf{1} \otimes \mathbf{1}) \diamond \Delta(v)=\Delta(u) \diamond \Delta(v) .
$$

Assume $u \neq \mathbf{1}$ and $v \neq \mathbf{1}$, and we proceed to prove this case by using induction on the sum of the depths

$$
n:=\operatorname{dep}(u)+\operatorname{dep}(v)
$$

For the initial step of $n=0$, we have $\operatorname{dep}(u)=\operatorname{dep}(v)=0$ and so $u, v \in M(X)$. Then the result is valid from Lemma 2.9 .

For the inductive step, assume the result holds for $n \leq k$ for a $k \geq 0$, and consider the case of $n=k+1$. We reduce to prove the result by induction on the sum of the widths

$$
m:=\operatorname{wid}(\mathrm{u})+\operatorname{wid}(\mathrm{v}) \geq 2 .
$$

If $m=2$, then $\operatorname{wid}(\mathrm{u})=\operatorname{wid}(\mathrm{v})=1$. Consider first $u \in X$ or $v \in X$. Then the result holds by Eq. (9). Consider next $u \notin X$ and $v \notin X$. Then by $\operatorname{wid}(\mathrm{u})=\operatorname{wid}(\mathrm{v})=1$, we can write

$$
u:=\lfloor\bar{u}\rfloor \text { and } v:=\lfloor\bar{v}\rfloor \text { for some } \bar{u}, \bar{v} \in \mathfrak{X}_{\infty} .
$$

Using the Sweedler notation, we denote by

$$
\Delta(\bar{u})=\sum_{(\bar{u})} \bar{u}_{(1)} \otimes \bar{u}_{(2)} \text { and } \Delta(\bar{v})=\sum_{(\bar{v})} \bar{v}_{(1)} \otimes \bar{v}_{(2)} .
$$

Thus we have

$$
\begin{aligned}
& \Delta(u \diamond v)=\Delta(\lfloor\bar{u}\rfloor \diamond\lfloor\bar{v}\rfloor)=\Delta(\lfloor\bar{u} \diamond\lfloor\bar{v}\rfloor+\lfloor\bar{u}\rfloor \diamond \bar{v}-\lfloor\bar{u} \diamond \bar{v}\rfloor\rfloor) \\
& =\left(\mathrm{id} \otimes N_{X}\right) \Delta(\bar{u} \diamond\lfloor\bar{v}\rfloor+\lfloor\bar{u}\rfloor \diamond \bar{v}-\lfloor\bar{u} \diamond \bar{v}\rfloor) \quad \text { (by Eq. ([8]) } \\
& \left.=\left(\mathrm{id} \otimes N_{X}\right)(\Delta(\bar{u}) \diamond \Delta(\lfloor\bar{v}\rfloor)+\Delta(\lfloor\bar{u}\rfloor) \diamond \Delta(\bar{v})-\Delta(\lfloor\bar{u} \diamond \bar{v}\rfloor)) \quad \text { (by the induction hypothesis on } n\right) \\
& \left.=\left(\mathrm{id} \otimes N_{X}\right)\left(\Delta(\bar{u}) \diamond\left(\left(\mathrm{id} \otimes N_{X}\right) \Delta(\bar{v})\right)+\left(\left(\mathrm{id} \otimes N_{X}\right) \Delta(\bar{u})\right) \diamond \Delta(\bar{v})-\left(\mathrm{id} \otimes N_{X}\right)(\Delta(\bar{u}) \diamond \Delta(\bar{v}))\right) \quad \text { (by Eq. ( }(8)\right) \\
& =\left(\mathrm{id} \otimes N_{X}\right)\left(\sum_{(\bar{u}),(\bar{v})}\left(\bar{u}_{(1)} \diamond \bar{v}_{(1)}\right) \otimes\left(\bar{u}_{(2)} \diamond\left\lfloor\bar{v}_{(2)}\right\rfloor\right)+\sum_{(\bar{u}),(\bar{v})}\left(\bar{u}_{(1)} \diamond \bar{v}_{(1)}\right) \otimes\left(\left\lfloor\bar{u}_{(2)}\right\rfloor \diamond \bar{v}_{(2)}\right)\right. \\
& \left.-\sum_{(\bar{u}),(\bar{v})}\left(\bar{u}_{(1)} \diamond \bar{v}_{(1)}\right) \otimes\left\lfloor\bar{u}_{(2)} \diamond \bar{v}_{(2)}\right\rfloor\right) \\
& =\sum_{(\bar{u}),(\bar{v})}\left(\bar{u}_{(1)} \diamond \bar{v}_{(1)}\right) \otimes\left(\left\lfloor\bar{u}_{(2)} \diamond\left\lfloor\bar{v}_{(2)}\right\rfloor\right\rfloor+\left\lfloor\left\lfloor\bar{u}_{(2)}\right\rfloor \diamond \bar{v}_{(2)}\right\rfloor-\left\lfloor\left\lfloor\bar{u}_{(2)} \diamond \bar{v}_{(2)}\right\rfloor\right\rfloor\right) \\
& \left.=\sum_{(\bar{u}),(\bar{v})}\left(\bar{u}_{(1)} \diamond \bar{v}_{(1)}\right) \otimes\left(\left\lfloor\bar{u}_{(2)}\right\rfloor \diamond\left\lfloor\bar{v}_{(2)}\right\rfloor\right) \quad \text { (by Eq. (2) }\right) \\
& =\left(\sum_{(\bar{u})} \bar{u}_{(1)} \otimes\left\lfloor\bar{u}_{(2)}\right\rfloor\right) \diamond\left(\sum_{(\bar{v})} \bar{v}_{(1)} \otimes\left\lfloor\bar{v}_{(2)}\right\rfloor\right) \\
& =\left(\left(\mathrm{id} \otimes N_{X}\right) \Delta(\bar{u})\right) \diamond\left(\left(\mathrm{id} \otimes N_{X}\right) \Delta(\bar{v})\right)
\end{aligned}
$$


$=\Delta(\lfloor\bar{u}\rfloor) \diamond \Delta(\lfloor\bar{v}\rfloor) \quad($ by Eq. $(\underline{8}))$

$=\Delta(u) \diamond \Delta(v)$.

This completes the initial step of the induction on $\operatorname{wid}(\mathrm{u})+\operatorname{wid}(\mathrm{v})$.

Assume that Eq. (10) holds for the case of $n=k+1$ and $2 \leq m \leq \ell$, and consider the case of $n=k+1$ and $m=\ell+1$. Then $m \geq 3$ and so either wid(u) $\geq 2$ or $\operatorname{wid}(\mathrm{v}) \geq 2$. We have three cases to prove:

(a) $\operatorname{wid}(\mathrm{u}) \geq 2, \operatorname{wid}(\mathrm{v}) \geq 2$;

(b) $\operatorname{wid}(\mathrm{u}) \geq 2, \operatorname{wid}(\mathrm{v})=1$;

(c) $\operatorname{wid}(\mathrm{u})=1, \operatorname{wid}(\mathrm{v}) \geq 2$.

We only prove the first case, because other cases are easier and can be treated in the same way. So suppose

$$
\operatorname{wid}(\mathrm{u}) \geq 2 \text { and } \operatorname{wid}(\mathrm{v}) \geq 2 .
$$

From Proposition 2.5, there are unique alternating sequences $u_{1}, \cdots, u_{p}$ and $v_{1}, \cdots, v_{q}$ such that

$$
u=u_{1} \diamond \cdots \diamond u_{p} \text { and } v=v_{1} \diamond \cdots \diamond v_{q} \text {, }
$$

and so

$$
u \diamond v=u_{1} \diamond \cdots \diamond u_{p-1} \diamond\left(u_{p} \diamond v_{1}\right) \diamond v_{2} \cdots \diamond v_{q}
$$

If $u_{p} \notin\left\lfloor\mathfrak{X}_{\infty}\right\rfloor$ or $v_{1} \notin\left\lfloor\mathfrak{X}_{\infty}\right\rfloor$, then by Eq. (9),

$$
\begin{aligned}
\Delta(u \diamond v) & =\Delta\left(u_{1} \diamond \cdots \diamond u_{p} \diamond v_{1} \diamond \cdots \diamond v_{q}\right) \\
& =\Delta\left(u_{1}\right) \diamond \cdots \diamond \Delta\left(u_{p}\right) \diamond \Delta\left(v_{1}\right) \diamond \cdots \diamond \Delta\left(v_{q}\right) \\
& =\Delta\left(u_{1} \diamond \cdots \diamond u_{p}\right) \diamond \Delta\left(v_{1} \diamond \cdots \diamond v_{q}\right) \\
& =\Delta(u) \diamond \Delta(v) .
\end{aligned}
$$

If $u_{p} \in\left\lfloor\mathfrak{X}_{\infty}\right\rfloor$ and $v_{1} \in\left\lfloor\mathfrak{X}_{\infty}\right\rfloor$, let

$$
u_{p}=\left\lfloor\bar{u}_{p}\right\rfloor \text { and } v_{1}=\left\lfloor\bar{v}_{1}\right\rfloor \text { for some } \bar{u}_{p}, \bar{v}_{1} \in \mathfrak{X}_{\infty} .
$$

By Eq. (2), we may suppose

$$
u_{p} \diamond v_{1}=\sum_{i} c_{i}\left\lfloor w_{i}\right\rfloor, \text { where each } c_{i} \in \mathbf{k} \backslash\{0\}, w_{i} \in \mathfrak{X}_{\infty} .
$$

So we get

$$
\begin{aligned}
\Delta(u \diamond v) & =\Delta\left(u_{1} \diamond \cdots \diamond u_{p-1} \diamond\left(u_{p} \diamond v_{1}\right) \diamond v_{2} \diamond \cdots \diamond v_{q}\right) \\
& =\sum_{i} c_{i} \Delta\left(u_{1} \diamond \cdots \diamond u_{p-1} \diamond\left\lfloor w_{i}\right\rfloor \diamond v_{2} \diamond \cdots \diamond v_{q}\right) \\
& \left.=\sum_{i} c_{i} \Delta\left(u_{1}\right) \diamond \cdots \diamond \Delta\left(u_{p-1}\right) \diamond \Delta\left(\left\lfloor w_{i}\right\rfloor\right) \Delta \Delta\left(v_{2}\right) \diamond \cdots \diamond \Delta\left(v_{q}\right) \quad \text { (by Eq. (9) }\right) \\
& =\Delta\left(u_{1}\right) \diamond \cdots \diamond \Delta\left(u_{p-1}\right) \diamond \Delta\left(u_{p} \diamond v_{1}\right) \diamond \Delta\left(v_{2}\right) \diamond \cdots \diamond \Delta\left(v_{q}\right) \\
& \left.=\Delta\left(u_{1}\right) \diamond \cdots \diamond \Delta\left(u_{p-1}\right) \diamond \Delta\left(u_{p}\right) \diamond \Delta\left(v_{1}\right) \diamond \Delta\left(v_{2}\right) \diamond \cdots \diamond \Delta\left(v_{q}\right) \quad \text { (by the case of } m=2\right) \\
& =\Delta\left(u_{1} \diamond \cdots \diamond u_{p}\right) \diamond \Delta\left(v_{1} \diamond \cdots \diamond v_{q}\right) \quad(\text { by Eq. (9) }) \\
& =\Delta(u) \diamond \Delta(v) .
\end{aligned}
$$

This completes the induction on $\operatorname{wid}(\mathrm{u})+\operatorname{wid}(\mathrm{v})=\mathrm{m}$ and so the induction on $\operatorname{dep}(u)+\operatorname{dep}(v)=$ $n$. 
Lemma 2.11. Let $X$ be a set. Then $\Delta$ is a coproduct on $F_{\mathrm{N}}(X)$, that is, $\Delta$ satisfies the coassociative law

$$
(\Delta \otimes \mathrm{id}) \Delta(w)=(\mathrm{id} \otimes \Delta) \Delta(w) \text { for } w \in F_{\mathrm{N}}(X) .
$$

Proof. We prove the result by induction on $\operatorname{depth} n:=\operatorname{dep}(w)$ of the basis elements $w \in \mathfrak{X}_{\infty}$. For the initial step of $n=0$, we have $w \in M(X)$. By Lemma 2.8, we get $\Delta(w)=\mathbf{1} \otimes w$ and so

$$
\begin{aligned}
(\mathrm{id} \otimes \Delta) \Delta(w) & =(\mathrm{id} \otimes \Delta)(\mathbf{1} \otimes w)=\mathbf{1} \otimes \Delta(w)=\mathbf{1} \otimes \mathbf{1} \otimes w \\
& =\Delta(\mathbf{1}) \otimes w=(\Delta \otimes \mathrm{id})(\mathbf{1} \otimes w)=(\Delta \otimes \mathrm{id}) \Delta(w) .
\end{aligned}
$$

For the inductive step, assume the result is true for the case of $n \leq k$ for a $k \geq 0$, and consider the case of $n=k+1$. We next reduce to prove the result by induction on the width $m:=\operatorname{wid}(\mathrm{w})$. Since $\operatorname{dep}(w)=k+1 \geq 1$, we have $w \neq \mathbf{1}$ and so $m=\operatorname{wid}(\mathrm{w}) \geq 1$. If $m=1$, since $\operatorname{dep}(w) \geq 1$, we can write

$$
w=\lfloor\bar{w}\rfloor=N_{X}(\bar{w}) \text { for some } \bar{w} \in \mathfrak{X}_{k} .
$$

So we get

$$
\begin{aligned}
(\mathrm{id} \otimes \Delta) \Delta(w) & =(\mathrm{id} \otimes \Delta) \Delta\left(N_{X}(\bar{w})\right) \\
& \left.=(\mathrm{id} \otimes \Delta)\left(\mathrm{id} \otimes N_{X}\right) \Delta(\bar{w}) \quad \text { (by Eq. (8) }\right) \\
& \left.=\left(\mathrm{id} \otimes\left(\Delta N_{X}\right)\right) \Delta(\bar{w}) \quad \text { by Eq. (5) }\right) \\
& =\left(\mathrm{id} \otimes\left(\left(\mathrm{id} \otimes N_{X}\right) \Delta\right)\right) \Delta(\bar{w}) \quad(\text { by Eq. (8) }) \\
& =\left(\mathrm{id} \otimes \mathrm{id} \otimes N_{X}\right)(\mathrm{id} \otimes \Delta) \Delta(\bar{w}) \\
& \left.=\left(\mathrm{id} \otimes \mathrm{id} \otimes N_{X}\right)(\Delta \otimes \mathrm{id}) \Delta(\bar{w}) \quad \text { (by the induction hypothesis on } n\right) \\
& =\left(\Delta \otimes N_{X}\right) \Delta(\bar{w}) \\
& =(\Delta \otimes \mathrm{id})\left(\mathrm{id} \otimes N_{X}\right) \Delta(\bar{w}) \\
& =(\Delta \otimes \mathrm{id}) \Delta(w) \quad(\text { by Eq. (8) }) .
\end{aligned}
$$

Assume that the result is valid for the case of $n=k+1$ and $m \leq \ell$, and consider the case of $n=k+1$ and $m=\ell+1 \geq 2$. So we can suppose

$$
w=w_{1}^{\prime} w_{2}^{\prime}=w_{1}^{\prime} \diamond w_{2}^{\prime},
$$

where

$$
\operatorname{wid}\left(\mathrm{w}_{1}^{\prime}\right)+\operatorname{wid}\left(\mathrm{w}_{2}^{\prime}\right)=\ell+1 \text { and } 1 \leq \operatorname{wid}\left(\mathrm{w}_{1}^{\prime}\right), \operatorname{wid}\left(\mathrm{w}_{2}^{\prime}\right) \leq \ell .
$$

Using the Sweedler notation, we can write

$$
\Delta\left(w_{1}^{\prime}\right)=\sum_{\left(w_{1}^{\prime}\right)} w_{1(1)}^{\prime} \otimes w_{1(2)}^{\prime} \text { and } \Delta\left(w_{2}^{\prime}\right)=\sum_{\left(w_{2}^{\prime}\right)} w_{2(1)}^{\prime} \otimes w_{2(2)}^{\prime} .
$$

By the induction hypothesis on $m$, we have

$$
(\Delta \otimes \mathrm{id}) \Delta\left(w_{1}^{\prime}\right)=(\mathrm{id} \otimes \Delta) \Delta\left(w_{1}^{\prime}\right) \text { and }(\Delta \otimes \mathrm{id}) \Delta\left(w_{2}^{\prime}\right)=(\mathrm{id} \otimes \Delta) \Delta\left(w_{2}^{\prime}\right),
$$

that is,

$$
\sum_{\left(w_{1}^{\prime}\right)} \Delta\left(w_{1(1)}^{\prime}\right) \otimes w_{1(2)}^{\prime}=\sum_{\left(w_{1}^{\prime}\right)} w_{1(1)}^{\prime} \otimes \Delta\left(w_{1(2)}^{\prime}\right) \text { and } \sum_{\left(w_{2}^{\prime}\right)} \Delta\left(w_{2(1)}^{\prime}\right) \otimes w_{2(2)}^{\prime}=\sum_{\left(w_{2}^{\prime}\right)} w_{2(1)}^{\prime} \otimes \Delta\left(w_{2(2)}^{\prime}\right) .
$$


So we get

$$
\begin{aligned}
(\mathrm{id} \otimes \Delta) \Delta(w) & =(\mathrm{id} \otimes \Delta) \Delta\left(w_{1}^{\prime} \diamond w_{2}^{\prime}\right) \\
& =(\mathrm{id} \otimes \Delta)\left(\Delta\left(w_{1}^{\prime}\right) \diamond \Delta\left(w_{2}^{\prime}\right)\right) \quad(\text { by Eq. (10) }) \\
& =\sum_{\left(w_{1}^{\prime}\right)} \sum_{\left(w_{2}^{\prime}\right)}\left(w_{1(1)}^{\prime} \diamond w_{2(1)}^{\prime}\right) \otimes \Delta\left(w_{1(2)}^{\prime} \diamond w_{2(2)}^{\prime}\right) \quad \text { (by linearity) } \\
& \left.=\sum_{\left(w_{1}^{\prime}\right)} \sum_{\left(w_{2}^{\prime}\right)}\left(w_{1(1)}^{\prime} \diamond w_{2(1)}^{\prime}\right) \otimes\left(\Delta\left(w_{1(2)}^{\prime}\right) \diamond \Delta\left(w_{2(2)}^{\prime}\right)\right) \quad \text { (by Eq. (10) }\right) \\
& =\left(\sum_{\left(w_{1}^{\prime}\right)} w_{1(1)}^{\prime} \otimes \Delta\left(w_{1(2)}^{\prime}\right)\right) \diamond\left(\sum_{\left(w_{2}^{\prime}\right)} w_{2(1)}^{\prime} \otimes \Delta\left(w_{2(2)}^{\prime}\right)\right) .
\end{aligned}
$$

With the similar argument, we can get

$$
(\Delta \otimes \mathrm{id}) \Delta(w)=\left(\sum_{\left(w_{1}^{\prime}\right)} \Delta\left(w_{1(1)}^{\prime}\right) \otimes w_{1(2)}^{\prime}\right) \diamond\left(\sum_{\left(w_{2}^{\prime}\right)} \Delta\left(w_{2(1)}^{\prime}\right) \otimes w_{2(2)}^{\prime}\right) .
$$

Thus Eq. (11) holds by Eq. (12). This completes the inductive proof.

We turn to construct a left counit on $F_{\mathrm{N}}(X)$. Define a linear map:

$$
\varepsilon: F_{\mathrm{N}}(X) \rightarrow \mathbf{k}, \mathbf{1} \mapsto 1_{\mathbf{k}}, w \mapsto 0 \text { for } w \in \mathfrak{X}_{\infty} \backslash\{\mathbf{1}\} .
$$

The next result shows that $\varepsilon$ is compatible with the product $\diamond$.

Lemma 2.12. Let $X$ be a set and $u, v \in F_{\mathrm{N}}(X)$. Then

$$
\varepsilon(u \diamond v)=\varepsilon(u) \varepsilon(v) .
$$

Proof. By linearity of $\varepsilon$, it is sufficient to prove the result for basis elements $u, v \in \mathfrak{X}_{\infty}$. If $u=\mathbf{1}$ or $v=\mathbf{1}$, by symmetry, let $u=\mathbf{1}$. Then $\varepsilon(u)=1_{\mathbf{k}}$ and

$$
\varepsilon(u \diamond v)=\varepsilon(v)=1_{\mathbf{k}} \varepsilon(v)=\varepsilon(u) \varepsilon(v) .
$$

Suppose $u \neq 1$ and $v \neq 1$. Then $u \diamond v \neq 1$ and so by Eq. (13)

$$
\varepsilon(u \diamond v)=0=\varepsilon(u) \varepsilon(v),
$$

as required.

Lemma 2.13. Let $X$ be a set. Then the $\varepsilon$ given in Eq. (13) is a left counit on $F_{\mathrm{N}}(X)$, that is, $\varepsilon$ satisfies the left counicity

$$
(\varepsilon \otimes \mathrm{id}) \Delta(w)=\beta_{\ell}(w) \text { for } w \in F_{\mathrm{N}}(X),
$$

where $\beta_{\ell}: F_{\mathrm{N}}(X) \rightarrow \mathbf{k} \otimes F_{\mathrm{N}}(X)$ is given by $w \mapsto 1_{\mathbf{k}} \otimes w$.

Proof. We prove the result by induction on $\operatorname{depth} n:=\operatorname{dep}(w)$ of basis elements $w \in \mathfrak{X}_{\infty}$. For the initial step of $n=0$, we have $w \in M(X)$. By Lemma 2.8, we obtain $\Delta(w)=\mathbf{1} \otimes w$ and so

$$
(\varepsilon \otimes \mathrm{id}) \Delta(w)=(\varepsilon \otimes \mathrm{id})(\mathbf{1} \otimes w)=\varepsilon(\mathbf{1}) \otimes w=1_{\mathbf{k}} \otimes w=\beta_{\ell}(F) .
$$

For the induction step, assume the result is true for the case of $n \leq k$ for a $k \geq 0$, and consider the case of $n=k+1$. We next reduce to prove the result by induction on the width $m:=\operatorname{wid}(\mathrm{w})$. 
Since $\operatorname{dep}(w)=k+1 \geq 1$, we have $w \neq \mathbf{1}$ and so $m=\operatorname{wid}(\mathrm{w}) \geq 1$. If $m=1$, since $\operatorname{dep}(w) \geq 1$, we can write

which implies

$$
w=N_{X}(\bar{w}) \text { for some } \bar{w} \in \mathfrak{X}_{k},
$$

$$
\begin{aligned}
(\varepsilon \otimes \mathrm{id}) \Delta(w) & =(\varepsilon \otimes \mathrm{id}) \Delta\left(N_{X}(\bar{w})\right) \\
& \left.=(\varepsilon \otimes \mathrm{id})\left(\mathrm{id} \otimes N_{X}\right) \Delta(\bar{w}) \quad \text { (by Eq. }(\underline{8})\right) \\
& =\left(\mathrm{id} \otimes N_{X}\right)(\varepsilon \otimes \mathrm{id}) \Delta(\bar{w}) \\
& =\left(\mathrm{id} \otimes N_{X}\right)\left(1_{\mathbf{k}} \otimes \bar{w}\right) \quad(\text { by the induction hypothesis on } n) \\
& =1_{\mathbf{k}} \otimes w=\beta_{\ell}(w) .
\end{aligned}
$$

Assume the result holds for the case of $n=k+1$ and $m \leq \ell$, and consider the case of $n=k+1$ and $m=\ell+1 \geq 2$. So we can write

$$
w=w_{1} \diamond w_{2},
$$

where

$$
\operatorname{wid}\left(\mathrm{w}_{1}\right)+\operatorname{wid}\left(\mathrm{w}_{2}\right)=\ell+1 \text { and } 1 \leq \operatorname{wid}\left(\mathrm{w}_{1}\right), \operatorname{wid}\left(\mathrm{w}_{2}\right) \leq \ell .
$$

Using the Sweedler notation, we may write

$$
\Delta\left(w_{1}\right)=\sum_{\left(w_{1}\right)} w_{1(1)} \otimes w_{1(2)} \text { and } \Delta\left(w_{2}\right)=\sum_{\left(w_{2}\right)} w_{2(1)} \otimes w_{2(2)} .
$$

From the induction on $m$, we get

$$
(\varepsilon \otimes \mathrm{id}) \Delta\left(w_{1}\right)=\beta_{\ell}\left(w_{1}\right) \text { and }(\varepsilon \otimes \mathrm{id}) \Delta\left(w_{2}\right)=\beta_{\ell}\left(w_{2}\right),
$$

that is,

$$
\sum_{\left(w_{1}\right)} \varepsilon\left(w_{1(1)}\right) \otimes w_{1(2)}=1_{\mathbf{k}} \otimes w_{1} \text { and } \sum_{\left(w_{2}\right)} \varepsilon\left(w_{2(1)}\right) \otimes w_{2(2)}=1_{\mathbf{k}} \otimes w_{2},
$$

which implies

$$
\begin{aligned}
(\varepsilon \otimes \mathrm{id}) \Delta(w) & =(\varepsilon \otimes \mathrm{id}) \Delta\left(w_{1} \diamond w_{2}\right) \\
& =(\varepsilon \otimes \mathrm{id})\left(\Delta\left(w_{1}\right) \diamond \Delta\left(w_{2}\right)\right) \quad(\text { by Eq. (10) }) \\
& =\sum_{\left(w_{1}\right),\left(w_{2}\right)} \varepsilon\left(w_{1(1)} \diamond w_{2(1)}\right) \otimes\left(w_{1(2)} \diamond w_{2(2)}\right) \\
& =\sum_{\left(w_{1}\right),\left(w_{2}\right)}\left(\varepsilon\left(w_{1(1)}\right) \varepsilon\left(w_{2(1)}\right)\right) \otimes\left(w_{1(2)} \diamond w_{2(2)}\right) \quad(\text { by Lemma 2.12) }) \\
& =\left(\sum_{\left(w_{1}\right)} \varepsilon\left(w_{1(1)}\right) \otimes w_{1(2)}\right)\left(\sum_{\left(w_{2}\right)} \varepsilon\left(w_{2(1)}\right) \otimes w_{2(2)}\right) \\
& =\left(1_{\mathbf{k}} \otimes w_{1}\right)\left(1_{\mathbf{k}} \otimes w_{2}\right) \quad(\text { by Eq. (14) }) \\
& =1_{\mathbf{k}} \otimes\left(w_{1} \diamond w_{2}\right)=1_{\mathbf{k}} \otimes w=\beta_{\ell}(w) .
\end{aligned}
$$

This completes the induction on the width wid(w) and hence the induction on the depth $\operatorname{dep}(w)$.

Now we put the pieces together to state our main result of this section. Define a linear map

$$
u: \mathbf{k} \rightarrow F_{\mathrm{N}}(X), 1_{\mathbf{k}} \mapsto \mathbf{1} .
$$


Theorem 2.14. Let $X$ be a set. Then the sextuple $\left(F_{\mathrm{N}}(X), \diamond, u, \Delta, \varepsilon, N_{X}\right)$ is a left counital cocycle bialgebra.

Proof. By Lemma2.3, the quadruple $\left(F_{\mathrm{N}}(X), \diamond, u, N_{X}\right)$ is an operated algebra. From Lemmas 2.11 and 2.13, the triple $\left(F_{\mathrm{N}}(X), \Delta, \varepsilon\right)$ is a left counital coalgebra. Finally, $\left(F_{\mathrm{N}}(X), \diamond, u, \Delta, \varepsilon, N_{X}\right)$ is a left counital cocycle bialgebra by Eq. (8) and Lemmas 2.10, 2.12,

\section{Left counital Hopf algebra structures on fREe NiJenhuis algebras}

This section is devoted to a left counital Hopf algebraic structure on a free Nijenhuis algebra $F_{\mathrm{N}}(X)$. All algebras considered in this section are assumed to be of characteristic zero. The following concepts are from [22, Definitions 4.1 and 4.4].

Definition 3.1. A left counital bialgebra $(H, m, u, \Delta, \varepsilon)$ is called a graded left counital bialgebra if there are k-modules $H^{(n)}, n \geq 0$, of $H$ such that
(a) $H=\bigoplus_{n=0}^{\infty} H^{(n)}$;
(b) $H^{(p)} H^{(q)} \subseteq H^{(p+q)}$;
(c) $\Delta\left(H^{(n)}\right) \subseteq\left(H^{(0)} \otimes H^{(n)}\right) \oplus\left(\bigoplus_{\substack{p+q=n \\ p>0, q>0}} H^{(p)} \otimes H^{(q)}\right)$ for $n \geq 0$.

Further $H$ is called connected if in addition $H^{(0)}=\operatorname{im} u(=\mathbf{k})$ and $\operatorname{ker} \varepsilon=\bigoplus_{n \geq 1} H^{(n)}$.

Let $A$ be an algebra and $C$ a left counital coalgebra. Denote $R:=\operatorname{Hom}(C, A)$. For $f, g \in R$, the convolution product of $f$ and $g$ can still be defined by

$$
f * g:=m_{A}(f \otimes g) \Delta_{C} .
$$

Definition 3.2. Let $H=(H, m, u, \Delta, \varepsilon)$ be a left counital bialgebra. Let $e:=u \varepsilon$.

(a) A linear map $S$ of $H$ is called a right antipode for $H$ if

$$
\operatorname{id}_{H} * S=e \text {. }
$$

(b) A left counital bialgebra with a right antipode is called a left counital right antipode Hopf algebra or simply a left counital Hopf algebra.

In setting of connected graded left counital bialgebras, right antipodes come for free.

Lemma 3.3. ([22, Theorem 4.6]) A connected graded left counital bialgebra is a left counital Hopf algebra.

There is no accident that $F_{\mathrm{N}}(X)$ is a connected graded left counital bialgebra, as we will soon see. For a $w \in \mathfrak{X}_{\infty}$, define the degree of $w$ to be

$$
\operatorname{deg}(w):=\operatorname{deg}_{X}(w)+\operatorname{deg}_{N_{X}}(w),
$$

where $\operatorname{deg}_{N_{X}}(w)\left(\right.$ resp. $\left.\operatorname{deg}_{X}(w)\right)$ denotes the number of occurrences of $N_{X}$ (resp. $\left.x \in X\right)$ in $w$. For example, $\operatorname{deg}(\lfloor x\rfloor)=2$ and $\operatorname{deg}(x\lfloor x\rfloor)=3$. Define

$$
F_{\mathrm{N}}^{(n)}:=\mathbf{k}\left\{w \in \mathfrak{X}_{\infty} \mid \operatorname{deg}(w)=n\right\} \text {, where } n \geq 0 \text {. }
$$

Then

$$
F_{\mathrm{N}}(X)=\bigoplus_{n=0}^{\infty} F_{\mathrm{N}}^{(n)}, F_{\mathrm{N}}^{(0)}=\mathbf{k} \text { and } N_{X}\left(F_{\mathrm{N}}^{(n)}\right) \subseteq F_{\mathrm{N}}^{(n+1)}
$$


Here the inclusion follows from $\operatorname{deg}\left(N_{X}(w)\right)=\operatorname{deg}(w)+1$ for $w \in \mathfrak{X}_{\infty}$. We will show below that the grading as above is compatible with the product $\diamond$ and coproduct $\Delta$ on $F_{\mathrm{N}}(X)$.

Lemma 3.4. Let $X$ be a set and $F_{\mathrm{N}}(X)$ the free Nijenhuis algebra on $X$. Then

$$
F_{\mathrm{N}}^{(p)} \diamond F_{\mathrm{N}}^{(q)} \subseteq F_{\mathrm{N}}^{(p+q)} \text { for all } p, q \geq 0
$$

Proof. Let $w \in F_{\mathrm{N}}^{(p)}$ and $w^{\prime} \in F_{\mathrm{N}}^{(q)}$ be basis elements in $\mathfrak{X}_{\infty}$. By Proposition 2.5, we may suppose

$$
w=w_{1} \diamond \cdots \diamond w_{m} \text { and } w^{\prime}=w_{1}^{\prime} \diamond \cdots \diamond w_{m^{\prime}}^{\prime}
$$

where $\operatorname{wid}(\mathrm{w})=\mathrm{m}, \operatorname{wid}\left(\mathrm{w}^{\prime}\right)=\mathrm{m}^{\prime}$ and $m, m^{\prime} \geq 0$. We prove the result by induction on the sum $s:=p+q \geq 0$. For the initial step of $s=0$, we have $p=q=0$ and so $w=w^{\prime}=\mathbf{1}$ by Eq. (16). Hence

$$
w \diamond w^{\prime}=\mathbf{1} \in F_{\mathrm{N}}^{(0)}=F_{\mathrm{N}}^{(p+q)} .
$$

For the induction step, assume the result is true for the case of $s \leq k$ for a $k \geq 0$, and consider the case of $s=k+1$. Under this assumption, we reduce to prove the result by induction on the sum of widths $t:=m+m^{\prime}$. Since $s=k+1 \geq 1$, we have $t=m+m^{\prime} \geq 1$. If $t=1$, then either $m=0$ and $m^{\prime}=1$ or $m=1$ and $m^{\prime}=0$. Without loss of generality, let $m=0$ and $m^{\prime}=1$. Then $w=\mathbf{1}, p=0$ and so

$$
w \diamond w^{\prime}=w^{\prime} \in F_{\mathrm{N}}^{(q)}=F_{\mathrm{N}}^{(p+q)} .
$$

Assume the result is valid for the case of $s=k+1$ and $t \leq \ell$, and consider the case of $s=k+1$ and $t=\ell+1 \geq 2$. We have three cases to consider

(a) $\operatorname{wid}(w) \geq 2, \operatorname{wid}\left(\mathrm{w}^{\prime}\right) \geq 2$;

(b) $\operatorname{wid}(w) \geq 2, \operatorname{wid}\left(\mathrm{w}^{\prime}\right)=1$;

(c) $\operatorname{wid}(w)=1, \operatorname{wid}\left(\mathrm{w}^{\prime}\right) \geq 2$.

Here we only supply the explicit proof of the first case, because other cases are easier and can be checked similarly. Suppose

$$
\operatorname{wid}(w) \geq 2 \text { and } \operatorname{wid}\left(\mathrm{w}^{\prime}\right) \geq 2 \text {, }
$$

and denote by

$$
\widetilde{w}:=w_{1} \diamond \cdots \diamond w_{m-1} \text { and } \widetilde{w}^{\prime}:=w_{2}^{\prime} \diamond \cdots \diamond w_{m^{\prime}}^{\prime}
$$

Then

$$
w=\widetilde{w} \diamond w_{m} \text { and } w^{\prime}=w_{1}^{\prime} \diamond \widetilde{w}^{\prime}
$$

and

$$
p=\operatorname{deg}(w)=\operatorname{deg}(\widetilde{w})+\operatorname{deg}\left(w_{m}\right) \text { and } q=\operatorname{deg}\left(w^{\prime}\right)=\operatorname{deg}\left(w_{1}^{\prime}\right)+\operatorname{deg}\left(\widetilde{w}^{\prime}\right) .
$$

By the associativity of the product $\diamond$ and Definition 2.4

$$
w \diamond w^{\prime}=\left(\widetilde{w} \diamond w_{m}\right) \diamond\left(w_{1}^{\prime} \diamond \widetilde{w}^{\prime}\right)=\widetilde{w} \diamond\left(w_{m} \diamond w_{1}^{\prime}\right) \diamond \widetilde{w}^{\prime}=\widetilde{w}\left(w_{m} \diamond w_{1}^{\prime}\right) \widetilde{w}^{\prime},
$$

which implies

$$
\begin{aligned}
& w \diamond w^{\prime}=\widetilde{w}\left(w_{m} \diamond w_{1}^{\prime}\right) \widetilde{w}^{\prime} \\
& \in \widetilde{w} F_{\mathrm{N}}^{\left(\operatorname{deg}\left(w_{m}\right)+\operatorname{deg}\left(w_{1}^{\prime}\right)\right)} \widetilde{w}^{\prime} \quad \text { (by induction hypothesis on } t \text { ) } \\
& \left.\subseteq F_{\mathrm{N}}^{\left(\operatorname{deg}(\widetilde{w})+\operatorname{deg}\left(w_{m}\right)+\operatorname{deg}\left(w_{1}^{\prime}\right)+\operatorname{deg}\left(\widetilde{w}^{\prime}\right)\right)} \quad \text { (by Eq. (15) }\right) \\
& =F_{\mathrm{N}}^{(p+q)} \quad \text { (by Eq. (18)) } \text {. }
\end{aligned}
$$

This finishes the induction on $t$ and hence the induction on $s$. 
Lemma 3.5. Let $X$ be a set and $F_{\mathrm{N}}(X)$ the free Nijenhuis algebra on $X$. Then

$$
\Delta\left(F_{\mathrm{N}}^{(n)}\right) \subseteq\left(F_{\mathrm{N}}^{(0)} \otimes F_{\mathrm{N}}^{(n)}\right) \oplus\left(\bigoplus_{\substack{p+q=n \\ p>0, q>0}} F_{\mathrm{N}}^{(p)} \otimes F_{\mathrm{N}}^{(q)}\right) \text { for all } n \geq 0 .
$$

Proof. Let $w \in \mathfrak{X}_{\infty}$ be a basis element of $F_{\mathrm{N}}(X)$. We proceed to prove the result by induction on $n=\operatorname{deg}(w) \geq 0$. For the initial step of $n=\operatorname{deg}(w)=0$, we have $w=\mathbf{1}$ and so by Eq. (5)

$$
\Delta(\mathbf{1})=\mathbf{1} \otimes \mathbf{1} \in F_{\mathrm{N}}^{(0)} \otimes F_{\mathrm{N}}^{(0)} .
$$

For the induction step, assume the result is true for the case of $n \leq k$ for a $k \geq 0$, and consider the case of $n=k+1$. Under this assumption, we reduce to prove the result by induction on the width $m=\operatorname{wid}(\mathrm{w})$. Since $n=\operatorname{deg}(w) \geq 1$, we have $w \neq \mathbf{1}$ and so $m=\operatorname{wid}(\mathrm{w}) \geq 1$.

If $m=1$, then either $w=x$ for some $x \in X$ or $w=N_{X}(\bar{w})$ for some $\bar{w} \in \mathfrak{X}_{\infty}$. If $w=x$ for some $x \in X$, then $n=\operatorname{deg}(w)=1$ and

$$
\Delta(w)=\mathbf{1} \otimes x \in F_{\mathrm{N}}^{(0)} \otimes F_{\mathrm{N}}^{(1)}=F_{\mathrm{N}}^{(0)} \otimes F_{\mathrm{N}}^{(n)} .
$$

If $w=N_{X}(\bar{w})$ for some $\bar{w} \in \mathfrak{X}_{\infty}$, then

$$
\operatorname{deg}(\bar{w})=\operatorname{deg}(w)-1=n-1=k
$$

Using the induction hypothesis on $n$, we get

$$
\Delta(\bar{w}) \in\left(F_{\mathrm{N}}^{(0)} \otimes F_{\mathrm{N}}^{(k)}\right) \oplus\left(\bigoplus_{\substack{p+q=k \\ p>0, q>0}} F_{\mathrm{N}}^{(p)} \otimes F_{\mathrm{N}}^{(q)}\right) .
$$

By Eqs. (8) and (16),

$$
\begin{aligned}
\Delta(w)=\Delta\left(N_{X}(\bar{w})\right) & =\left(\mathrm{id} \otimes N_{X}\right) \Delta(\bar{w}) \\
& \in\left(F_{\mathrm{N}}^{(0)} \otimes N_{X}\left(F_{\mathrm{N}}^{(k)}\right)\right) \oplus\left(\bigoplus_{\substack{p+q=k \\
p>0, q>0}} F_{\mathrm{N}}^{(p)} \otimes N_{X}\left(F_{\mathrm{N}}^{(q)}\right)\right) \\
& \subseteq\left(F_{\mathrm{N}}^{(0)} \otimes F_{\mathrm{N}}^{(k+1)}\right) \oplus\left(\bigoplus_{\substack{p+q=k+1 \\
p>0, q>1}} F_{\mathrm{N}}^{(p)} \otimes F_{\mathrm{N}}^{(q)}\right) \\
& \subseteq\left(F_{\mathrm{N}}^{(0)} \otimes F_{\mathrm{N}}^{(k+1)}\right) \oplus\left(\bigoplus_{\substack{p+q=k+1 \\
p>0, q>0}} F_{\mathrm{N}}^{(p)} \otimes F_{\mathrm{N}}^{(q)}\right) .
\end{aligned}
$$

Assume the result holds for the case of $n=k+1$ and $m \leq \ell$, and consider the case of $n=k+1$ and $m=\ell+1 \geq 2$. From Proposition 2.5, we may write

$$
w=u \diamond v, \text { where } u, v \in \mathfrak{X}_{\infty} \text { and } 0<\operatorname{wid}(\mathrm{u}), \operatorname{wid}(\mathrm{v})<\ell+1 \text {. }
$$

Using the induction hypothesis on $m$, we get

$$
\Delta(u) \in\left(F_{\mathrm{N}}^{(0)} \otimes F_{\mathrm{N}}^{(\operatorname{deg}(u))}\right) \oplus\left(\bigoplus_{\substack{p+q=\operatorname{deg}(u) \\ p>0, q>0}} F_{\mathrm{N}}^{(p)} \otimes F_{\mathrm{N}}^{(q)}\right)
$$

and

$$
\Delta(v) \in\left(F_{\mathrm{N}}^{(0)} \otimes F_{\mathrm{N}}^{(\operatorname{deg}(v))}\right) \oplus\left(\bigoplus_{\substack{p^{\prime}+q^{\prime}=\operatorname{deg}(v) \\ p^{\prime}>0, q^{\prime}>0}} F_{\mathrm{N}}^{\left(p^{\prime}\right)} \otimes F_{\mathrm{N}}^{\left(q^{\prime}\right)}\right)
$$


Since $w=u \diamond v=u v$, we have

$$
\operatorname{deg}(u)+\operatorname{deg}(v)=\operatorname{deg}(w)=n=k+1 .
$$

Thus we obtain

$$
\begin{aligned}
\Delta(w)= & \Delta(u) \diamond \Delta(v) \\
\in & \left.\left(\left(F_{\mathrm{N}}^{(0)} \otimes F_{\mathrm{N}}^{(\operatorname{deg}(u))}\right) \oplus\left(\bigoplus_{\substack{p+q=\operatorname{deg}(u) \\
p>0, q>0}} F_{\mathrm{N}}^{(p)} \otimes F_{\mathrm{N}}^{(q)}\right)\right) \diamond\left(F_{\mathrm{N}}^{(0)} \otimes F_{\mathrm{N}}^{(\operatorname{deg}(v))}\right) \oplus\left(\bigoplus_{\substack{p^{\prime}+q^{\prime}=\operatorname{deg}(v) \\
p^{\prime}>0, q^{\prime}>0}} F_{\mathrm{N}}^{\left(p^{\prime}\right)} \otimes F_{\mathrm{N}}^{\left(q^{\prime}\right)}\right)\right) \\
= & \left(F_{\mathrm{N}}^{(0)} \diamond F_{\mathrm{N}}^{(0)}\right) \otimes\left(F_{\mathrm{N}}^{(\operatorname{deg}(u))} \diamond F_{\mathrm{N}}^{(\operatorname{deg}(v))}\right) \oplus\left(\bigoplus_{\substack{p^{\prime}+q^{\prime}=\operatorname{deg}(v) \\
p^{\prime}>0, q^{\prime}>0}}\left(F_{\mathrm{N}}^{(0)} \diamond F_{\mathrm{N}}^{\left(p^{\prime}\right)}\right) \otimes\left(F_{\mathrm{N}}^{(\operatorname{deg}(u))} \diamond F_{\mathrm{N}}^{\left(q^{\prime}\right)}\right)\right) \\
& \oplus\left(\bigoplus_{\substack{p+q=\operatorname{deg}(u) \\
p>0, q>0}}\left(F_{\mathrm{N}}^{(p)} \diamond F_{\mathrm{N}}^{(0)}\right) \otimes\left(F_{\mathrm{N}}^{(q)} \diamond F_{\mathrm{N}}^{(\operatorname{deg}(v))}\right)\right) \\
& \left(\bigoplus_{\substack{p+q=\operatorname{deg}(u), p^{\prime}+q^{\prime}=\operatorname{deg}(v) \\
p>0, q>0, p^{\prime}>0, q^{\prime}>0}}\left(F_{\mathrm{N}}^{(p)} \diamond F_{\mathrm{N}}^{\left(p^{\prime}\right)}\right) \otimes\left(F_{\mathrm{N}}^{(q)} \diamond F_{\mathrm{N}}^{\left(q^{\prime}\right)}\right)\right) \\
\subseteq & \left(F_{\mathrm{N}}^{(0)} \otimes F_{\mathrm{N}}^{(n)}\right) \oplus\left(\bigoplus_{\substack{p+q=n \\
p>0, q>0}} F_{\mathrm{N}}^{(p)} \otimes F_{\mathrm{N}}^{(q)}\right) \quad(\text { by Eqs. (17) and } \underline{190)}) .
\end{aligned}
$$

This completes the induction on wid(w) and hence the induction on $\operatorname{deg}(w)$.

Now we arrive at the main result of the paper.

Theorem 3.6. Let $X$ be a set and $F_{\mathrm{N}}(X)$ the free Nijenhuis algebra on $X$. Then the quintuple $\left(F_{\mathrm{N}}(X), \diamond, N_{X}, \Delta, \varepsilon\right)$ is a connected graded left counital bialgebra and hence a left counital Hopf algebra.

Proof. The $\left(F_{\mathrm{N}}(X), \diamond, N_{X}, \Delta, \varepsilon\right)$ is a left counital bialgebra by Theorem 2.14, and further is graded by Eq. (16) and Lemmas 3.4, 3.5, The connectedness follows from Eqs. (13) and (16). Therefore the result is valid from Lemma 3.3 .

Acknowledgements: This work was supported by the National Natural Science Foundation of China (Grant No. 11501466), Fundamental Research Funds for the Central Universities (Grant No. lzujbky-2017-162), and the Natural Science Foundation of Gansu Province (Grant No. 17JR5 RA175) and Shandong Province (No. ZR2016AM02).

We thank the anonymous referee for valuable suggestions helping to improve the paper.

\section{REFERENCES}

[1] E. Abe, Hopf Algebras, Cambridge University Press, 1980.

[2] G. E. Andrews, L. Guo, W. Keigher and K. Ono, Baxter algebras and Hopf algebras, Trans. Amer. Math. Soc. 355 (2003), 4639-4656.

[3] G. Baxter, An analytic problem whose solution follows from a simple algebraic identity, Pacific J. Math. 10 (1960), 731-742.

[4] A. A. Belavin and V. G. Drinfeld, Triangle Equations and Simple Lie-Algebras, Classic Reviews in Mathematics and Mathematical Physics, 1. Harwood Academic Publishers, Amsterdam, 1998, vii, 91 pp. 
[5] J. Cariñena, J. Grabowski and G. Marmo, Quantum bi-Hamiltonian systems, Internat. J. Modern Phys. A, 15, (2000), 4797-4810.

[6] A. Connes and D. Kreimer, Hopf algebras, renormalization and non-commutative geometry, Comm. Math. Phys. 199 (1998), 203-242.

[7] K. Ebraihimi-Fard, On the associative Nijenhuis Relation, Electrical J. Combin. 11(1) (2004), R38.

[8] A. Frölicher and A. Nijenhuis, Theory of vector valued differential forms. Part I, Indag. Math. 18 (1956), 338-360.

[9] X. Gao, L. Guo, W. Sit and S. Zheng, Rota-Baxter type operators, rewriting systems and GröbnerShirshov bases, J. Symbolic Computation, to appear, arXiv:1412.8055v1.

[10] I. Z. Golubchik and V. V. Sokolov, One more type of classical Yang-Baxter equation, Funct. Anal. Appl. 34 (2000), 296-298.

[11] I.Z. Golubchik and V. V. Sokolov, Generalized Operator Yang-Baxter Equations, Integrable ODEs and Nonassociative Algebras, J. of Nonlinear Math. Phys. 7 (2000), 184-197.

[12] L. Guo and W. Keigher, Baxter algebras and shuffle products, Adv. in Math. 150 (2000), 117-149.

[13] L. Guo, An Introduction to Rota-Baxter Algebra, International Press, 2012.

[14] L. Guo, Operated semigroups, Motzkin paths and rooted trees, J. Algebraic Combinatorics 29 (2009), $35-62$.

[15] Y. Kosmann-Schwarzbach and F. Magri, Poisson-Nijenhuis structures, Ann. Inst. Henri Poincaré 53 (1990), 35-81.

[16] P. Lei and L. Guo, Nijenhuis algebras, NS algebras and N-dendriform algebras, Frontiers of Mathematics in China 7(5), 2012, 827-846.

[17] J. L. Loday, M. O. Ronco, Trialgebras and families of polytopes, in "Homotopy Theory: Relations with algebraic Geometry, Group Cohomology, and Algebraic k-theory", Contemporary Mathematics 346 (2004), 369-398.

[18] A. Nijenhuis, $X_{n-1}$-forming sets of eigenvectors. Indag. Math. 13 (1951) 200-212.

[19] M. Sweedler, Hopf Algebras, Benjamin, New York, 1969.

[20] K. Uchino, Twisting on associative algebras and Rota-Baxter type operators, J. Noncommut. Geom. 4 (2010) 349-379.

[21] T. Zhang, X. Gao and L. Guo, Hopf algebras of rooted forests, cocyles and free Rota-Baxter algebras, J. Math. Phys. 57 (2016) 101701, arXiv:1605.09531.

[22] S. Zheng and L. Guo, Left counital Hopf algebra structure on free commutative Nijenhus algebras, arXiv:1711.04823v1.

School of Mathematics and Statistics, Key Laboratory of Applied Mathematics and Complex Systems, Lanzhou University, Lanzhou, Gansu 730000, P. R. China

E-mail address: gaoxing@lzu.edu.cn

School of Mathematics and Statistics, Key Laboratory of Applied Mathematics and Complex Systems, Lanzhou University, Lanzhou, Gansu 730000, P. R. China

E-mail address: leip@lzu.edu.cn

School of Mathematics and Statistics, Ningxia University, Yinchuan, Ningxia 750021, P. R. China

E-mail address: tjzhangmath@nxu. edu.cn 